\title{
Characterization of ventricular assist device-mediated sensitization in the bridge-to-heart-transplantation patient
}

\author{
Murray H. Kwon, MD, ${ }^{a}$ Jennifer Q. Zhang, PhD, ${ }^{b}$ Joanna M. Schaenman, MD, PhD, ${ }^{c}$ \\ Martin Cadeiras, MD, ${ }^{\mathrm{d}}$ David W. Gjertson, PhD, ${ }^{\mathrm{b}}$ Carolyn A. Krystal, ${ }^{\mathrm{e}}$ Hillel Laks, MD, ${ }^{\mathrm{a}}$ \\ Abbas Ardehali, MD, ${ }^{a}$ Mario C. Deng, MD, ${ }^{d}$ Richard J. Shemin, MD, ${ }^{a}$ and Elaine F. Reed, $\mathrm{PhD}^{\mathrm{b}}$
}

\begin{abstract}
Objective: Ventricular assist devices (VADs) are associated with increased anti-human leukocyte antigen antibody production. The purpose of this study is to characterize differences in sensitization patterns in patients receiving axial flow, implantable VADs versus pulsatile, paracorporeal biventricular assist devices (BIVADs) as bridges to transplantation.
\end{abstract}

Methods: The study is a retrospective review of 68 patients who were bridged to transplantation with either a VAD or a BIVAD, as described, from January 2007 to June 2010, at a university medical center.

Results: Five of $15(33.3 \%)$ VAD patients became sensitized during treatment, compared with 30 of $53(56.6 \%)$ BIVAD patients, $P=.15$. Multivariable analysis comparing BIVAD with VAD, while controlling for previous cardiac surgery, pregnancy, and packed red blood cell transfusion produced an odds ratio of $2.99, P=.14$. Of sensitized patients, all $5(100 \%)$ of the VAD patients had pre-existing antibodies before VAD placement, compared with 9 of $30(30.0 \%)$ BIVAD patients, $P=.006$. Maximum cumulative mean fluorescence intensities for BIVAD were $46,259 \pm 66,349$ versus $42,540 \pm 12,840$ for VAD, $P=.90$. Time to maximum antibody expression was shorter for the VAD group $(34 \pm 28$ days vs $5.8 \pm 9$ days, $P=.04)$.

Conclusions: Device type was not a factor in patient sensitization after implantation. However, VAD patients required pre-existing sensitization before implantation to produce antibodies during their treatment interval, whereas more than two thirds of BIVAD patients developed de novo antibodies. These data suggest that the mechanism of sensitization between VAD and BIVAD patients may differ, and further mechanistic studies into the impact of device types on patient sensitization are warranted. (J Thorac Cardiovasc Surg 2015;149:1161-6)

See related commentary on pages $1166-7$.

The presence of circulating anti-human leukocyte antigen (HLA) antibodies, or their sensitization, in heart transplant recipients is associated with decreased survival, increased episodes of acute cellular and antibody-mediated rejection, and increased development of allograft vasculopathy. ${ }^{1-3}$ Multiparity, previous cardiac surgery, and history of blood transfusions are the most commonly implicated etiologies. Recently, however, ventricular assist devices (VADs), commonly used as bridges to transplantation (BTTs) in

\footnotetext{
From the Division of Cardiothoracic Surgery, ${ }^{\mathrm{a}}$ Department of Surgery, David Geffen School of Medicine at the University of California, Los Angeles, Los Angeles; the University of California, Los Angeles Immunogenetics Center, ${ }^{\mathrm{b}}$ University of California, Los Angeles, Los Angeles; the Divisions of Immunology ${ }^{\mathrm{c}}$ and Cardiology, ${ }^{\mathrm{d}}$ Department of Medicine, David Geffen School of Medicine at the University of California, Los Angeles, Los Angeles, Calif; and the David Geffen School of Medicine at the University of California, ${ }^{\mathrm{e}}$ Los Angeles, Los Angeles, Calif.

Disclosures: Authors have nothing to disclose with regard to commercial support.

Received for publication Oct 17, 2014; revisions received Dec 19, 2014; accepted for publication Jan 2, 2015; available ahead of print Feb 19, 2015.

Address for reprints: Murray H. Kwon, MD, David Geffen School of Medicine at UCLA, 10833 Le Conte Avenue, CHS 62-229, Los Angeles, CA 90095 (E-mail: mkwon@mednet.ucla.edu).

$0022-5223 / \$ 36.00$

Copyright (C) 2015 by The American Association for Thoracic Surgery

http://dx.doi.org/10.1016/j.jtcvs.2015.01.003
}

the sickest orthotopic heart transplant candidates, are increasingly associated with the increased expression of circulating HLA antibodies. ${ }^{4,5}$

One important proposed etiology is host immune-cell interactions with the surfaces of the respective devices. This possibility is consistent with data showing that the latest generation of axial flow pumps, such as the HeartMate II left VAD (HMII) (Thoratec Corporation, Pleasanton, Calif), lead to lower rates of sensitization ( $8 \%$ vs $28 \%, P=.02)$ than their older, pulsatile counterparts, such as the paracorporeal biventricular assist device (BIVAD) or the HeartMate XVE (both from Thoratec Corporation, Pleasanton, Calif). ${ }^{6}$ The older pumps have bigger chamber surface areas and valves, whereas the HMII relies on a spinning rotor to propel blood in continuous fashion through a relatively small channel. The aim of the present study is to characterize the sensitization patterns for BTT patients undergoing HMII versus BIVAD implantations in our institution.

\section{METHODS}

Records for 68 patients, between the ages of 18 and 70 years, undergoing VAD insertion as a BTT, between January 2007 and June 2010, were retrospectively reviewed with approval of the UCLA (University of California, Los Angeles) Institutional Review Board. Patients were evaluated for previous cardiac surgery, pregnancy, and blood-product utilization during the VAD support interval. Patient sera samples were collected according to the existing clinical protocols at our institution and analyzed for antibodies directed against HLA class I (A, B, and C) and class II (DR, 


\section{Abbreviations and Acronyms \\ BIVAD $=$ biventricular assist device \\ BTT = bridge to transplantation \\ HLA = human leukocyte antigen \\ HMII = HeartMate II left ventricular assist device \\ $\mathrm{VAD}=$ ventricular assist device}

DQ, and DP) antigens utilizing Luminex reagents (Gen-Probe, San Diego, Calif) according to manufacturer specifications and antibody specificity reagents according to manufacturer specifications. Particle fluorescence was measured using the Luminex 100 IS system (Luminex Corporation, Austin, Tex). Additional Luminex-based single-antigen bead assays (One Lambda Inc, Canoga Park, Calif) were run on positive sera to confirm the antibody specificity and strength as indicated by the mean fluorescence intensity. Antibodies were considered positive when these intensity values were $\geq 1000$ for HLA-A, -B, -DR, -DQ, and -DP and $\geq 2000$ for HLA-C. ${ }^{7}$ The maximum value was determined by the selection of the sample date with the highest total summed mean fluorescence intensity values.

Device selection was made by a multidisciplinary team that included a cardiac surgeon and cardiologist. Patients were categorized as having INTERMACS (Interagency Registry for Mechanically Assisted Circulatory Support) level 1 or 2 heart failure with impending multiorgan failure and/or death from malperfusion. In the setting of isolated left-ventricular failure, the axial flow HMII was utilized. Temporary CentriMag centrifugal right VAD support was used when appropriate (Thoratec Corporation, Pleasanton, Calif). Profound biventricular failure prompted paracorporeal VAD placement in the right and left ventricles, respectively. All right VADs placed in the BIVAD group were thus permanent and remained in place until the time of orthotopic heart transplantation. Both the HMII and paracorporeal BIVADs are produced by Thoratec Corporation (Pleasanton, Calif) and are approved by the U.S. Food and Drug Administration for BTT indications.

\section{Statistical Analysis}

Calculated panel reactive antibody percentages were calculated, entering all unacceptable antigens for HLA-A, -B, -C, -DR, and -DQ, defined as those with signal strength mean fluorescence intensity $\geq 1000$ in the UNet computer system at the U.S. Department of Health \& Human Services Organ Procurement and Transplantation Network website (http:// optn.transplant.hrsa.gov). Noncontinuous variables were analyzed using $\chi 2$ analysis and the Student $t$ test. Continuous variables were compared using analysis of variance with Bonferroni correction. Multivariable regression analysis was performed to quantify the association between sensitization etiologies and outcomes.

\section{RESULTS}

Of 68 patients, BIVADs were placed in 53, and HMIIs were placed in the remaining 15 . A total of $56(82 \%)$ patients were men. Etiologies of heart failure were idiopathic dilated cardiomyopathy in $30(44 \%)$, ischemic cardiomyopathy in $26(38 \%)$, postpartum cardiomyopathy in $3(4 \%)$, and "other" in $9(13 \%)$. The average age of VAD recipients was $52 \pm 11.7$ years. Differences in history of cardiac surgery, pregnancy, and blood-product utilization between the BIVAD and HMII groups are shown in Table 1. Only fresh frozen plasma administration differed significantly between the 2 groups.

Multivariable analysis comparing development of HLA antibodies in BIVAD versus HMII patients, while controlling for each of these variables, demonstrated an odds ratio of 2.99 (95\% confidence interval 0.71-12.6), $P=.14$. Five of $15(33.3 \%)$ HMII patients produced anti-HLA antibodies during their VAD treatment intervals, compared with 30 of $53(56.6 \%)$ BIVAD patients $(P=.15)$. Table 2 shows common etiologies for patient sensitization, of which only packed red blood cell transfusion differed significantly between the sensitized and nonsensitized groups.

Of sensitized patients, all $5(100 \%)$ of the HMII patients had pre-existing antibodies before VAD placement, compared with 9 of $30(30.0 \%)$ of the BIVAD patients, $P=.006$ (Figure 1). Thus, all HMII patients who expressed anti-HLA antibodies had evidence of presensitization, whereas more than two thirds of BIVAD patients developed de novo antibodies during their VAD treatment course. Representative patterns of sensitization are shown in Figure 2, for both presensitized individuals (top) and patients who became sensitized after device placement (bottom). Two of the HMII patients had temporary right VADs, from which they were weaned before orthotopic heart transplantation. Neither patient became sensitized during their VAD treatment course.

Single-antigen bead assays were compared to determine HLA class I and II expression in patients with BIVADs versus HMIIs. Figure 3 shows that no HMII patients expressed class II antibodies alone, in contrast to $13.8 \%$ of the BIVAD patients in this group. A total of $51.7 \%$ of the BIVAD patients had just class I, compared with $80 \%$ of the HMII patients. The BIVAD and HMII patients expressing both class I and class II antibodies were $34.5 \%$ and $20 \%$, respectively $(P=.81)$.

The mean of the maximum mean fluorescence intensity values for class I antibodies for BIVADs was 46,422 \pm 66,264 versus $42,540 \pm 12,840$ for HMII, $P=.90$. Time to maximum antibody expression was shorter for the HMII group (5.8 \pm 8.6 days vs $33.8 \pm 27.8$ days, $P=.04$ ). With regard to class II antibodies, BIVADs reached a maximum of $29,937 \pm 31,468$ at a mean of 30.7 days, whereas the single HMII patient who had expression of class II antibodies had a maximum mean intensity value of 1499 at 19 days (Table 3 ).

To gauge the breadth of antibody specificities, we calculated the mean panel reactive antibody percentages for sensitized patients in both the HMII and BIVAD groups (Table 4). The mean initial percentage was significantly higher in the HMII group, compared with the BIVAD patients; however, the mean maximum percentage level was essentially equivalent between the 2 categories. Thus, the percentage change in the calculated panel reactive antibody percentages was significantly higher in the BIVAD group during the VAD treatment interval $(34.1 \% \pm 31.4 \%$ vs $4.0 \% \pm 7.9 \%$, respectively, $P=.045$ ).

To validate the findings of this study, we examined an additional 24 patients who had HMIIs put in place at our institution between July 2010 and December 2013. Nine of these patients produced HLA antibodies during their 
TABLE 1. Risk factors for sensitization in BIVAD and HMII patients

\begin{tabular}{lccr}
\hline & BIVAD & HMII & $\boldsymbol{P}$ value \\
\hline Previous cardiac surgery & 17.0 & 20.0 & .72 \\
Pregnancy & 11.3 & 13.3 & 1.00 \\
Packed red blood cells & $48.3 \pm 30.9$ & $32.3 \pm 29.8$ & .08 \\
Fresh frozen plasma & $29.1 \pm 15.2$ & $17.7 \pm 13.2$ & .01 \\
Platelets & $12.2 \pm 22.4$ & $4.9 \pm 5.1$ & .22 \\
Cryoprecipitate & $4.2 \pm 6.1$ & $3.6 \pm 3.0$ & .71 \\
\hline
\end{tabular}

Blood products are expressed as total units administered $( \pm$ SD) between mechanical circulatory support device implantation and orthotopic heart transplantation. Previous cardiac surgery and pregnancy values are $\%$. BIVAD, Biventricular assist device; HMII, HeartMate II left ventricular assist device.

BTT-period VAD treatment courses, of whom 7 (77.8\%) had pre-existing antibodies. During this same time period, 13 patients needed BIVADs, 5 of whom developed antibodies during their VAD treatment course. Four of these 5 patients had pre-existing antibodies. When these patients were added to the original cohort, a total of 14 in the resulting cohort were sensitized HMII patients, of whom 12 $(85.7 \%)$ had pre-existing antibodies, compared with 35 sensitized BIVAD patients, of whom only $13(37.1 \%)$ had antibodies at the onset of treatment $(P=.004)$. Thus, our study continues to validate the finding that the majority of sensitized BIVAD patients develop their antibodies de novo, compared with the HMII patients, among whom most of the sensitized patients had pre-existing antibodies.

\section{DISCUSSION}

According to a recent survey of 23 centers, $7.8 \%$ of patients transplanted from January 2000 to April 2008 were sensitized (362 of 4640), of whom 141 (39\%) were bridged to transplant with VADs. ${ }^{1}$ In a separate study, $66 \%$ of pretransplant patients supported with VADs were sensitized, as defined by the development of immunoglobulin $G$ antibodies to HLA antigens. ${ }^{8}$ This mode of sensitization occurred independently of blood transfusions and seemed to be affected by host interactions with the biological surfaces of the respective devices. ${ }^{6}$ Thus, in addition to the traditional etiologies of patient sensitization, such as

TABLE 2. Risk factors for sensitization in patients who develop HLA antibodies during their VAD course, compared with those who do not

\begin{tabular}{lccc}
\hline & $\begin{array}{c}\text { HLA } \\
\text { antibodies }+\end{array}$ & $\begin{array}{c}\text { HLA } \\
\text { antibodies }-\end{array}$ & $\begin{array}{c}\boldsymbol{P} \\
\text { value }\end{array}$ \\
\hline Previous cardiac surgery & 23.5 & 8.8 & .19 \\
Pregnancy & 20.6 & 2.9 & .05 \\
Packed red blood cells & $52.1 \pm 34.7$ & $35.9 \pm 22.3$ & .04 \\
Fresh frozen plasma & $29.4 \pm 14.4$ & $23.6 \pm 15.2$ & .15 \\
Platelets & $9.8 \pm 9.7$ & $6.8 \pm 6.7$ & .20 \\
Cryoprecipitate & $3.2 \pm 2.6$ & $3.1 \pm 2.6$ & .74 \\
\hline
\end{tabular}

Blood products are expressed as total units administered ( \pm SD) between mechanical circulatory support device implantation and orthotopic heart transplantation. Previous cardiac surgery and pregnancy values are $\%$. $H L A$, Human leukocyte antigen.

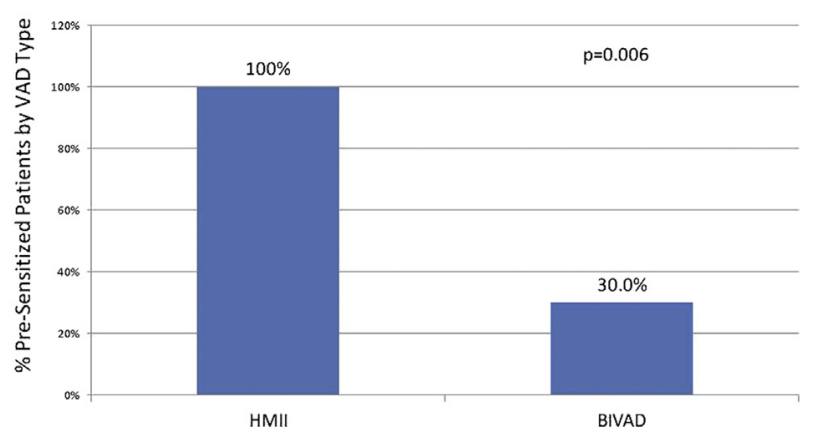

FIGURE 1. Percentage of sensitized patients who had pre-existing antibodies before VAD placement. A total of $100 \%$ of patients with sensitization in the HMII group had pre-existing antibodies, whereas only $38 \%$ of the BIVAD patients had similar findings. Thus, $62 \%$ of sensitized BIVAD patients developed their antibodies de novo after VAD insertion. $V A D$, Ventricular assist device; HMII, HeartMate II left ventricular assist device; $B I V A D$, biventricular assist device.

previous blood transfusions and multiparity, VAD therapy has emerged as an important causal agent for the development of HLA antibodies in the pretransplant patient.

The importance of the effect of biosurfaces may be seen in comparisons between the newer-generation axial flow devices, such as the HMII, and the older, volumedisplacement or pulsatile BIVADs. Although the former are thought to be less sensitizing, owing to their substantially smaller inner surface areas and lack of chamber
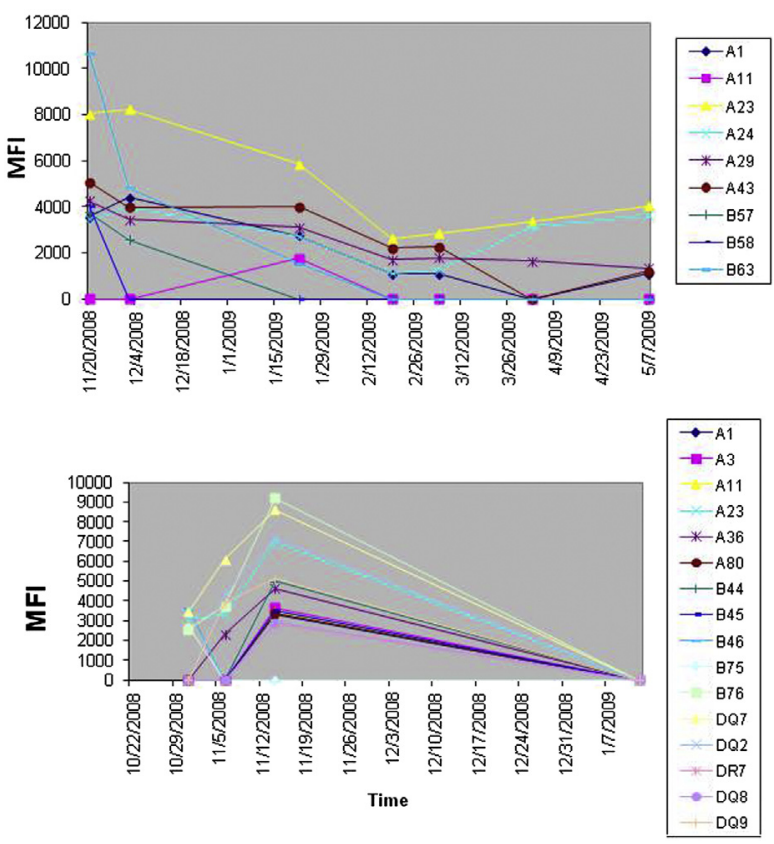

FIGURE 2. Representative pattern of sensitization post-VAD insertion for patients with pre-existing HLA antibodies (top) and those who formed their antibodies in de novo fashion after device placement (bottom). MFI, Mean fluorescence intensity. 


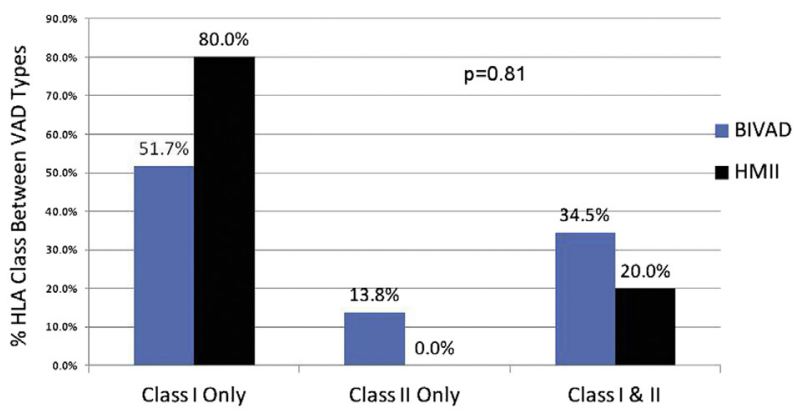

FIGURE 3. Frequencies of HLA class I and class II antibodies in sensitized patients by device type. $H L A$, Human leukocyte antigen; BIVAD, biventricular assist device; $H M I I$, HeartMate II left ventricular assist device; $V A D$, ventricular assist device.

valves, these characteristics have not been universally observed, as 1 group has shown disparate, higher rates of sensitization in up to $59 \%$ of patients with the smaller axial flow pumps. ${ }^{9}$

Sensitization mediated by a VAD may be secondary to the inflammatory effects of the biomaterials on circulating B cells, with the resulting dysregulated immunoglobulin synthesis. Studies of the cells that were detached from the textured neointimal surfaces of VADs detected monocyte/ macrophage lineages, as well as activated $\mathrm{T}$ lymphocytes secreting the cytokines interleukin (IL)-1, IL-2, and IL$10 .{ }^{10}$ Polyclonal B-cell activation is postulated to occur from these circulating helper T-cell cytokines, leading to increased expression of circulating anti-HLA antibodies in VAD patients compared with New York Heart Association class IV patients awaiting orthotopic heart transplantation without device support. ${ }^{11}$

The use of smaller devices, such as the HMII axial flow pump, is now favored, owing to their efficacy and better patient tolerance when compared with larger, oldergeneration pumps. No long-term biventricular axial flow device options are currently available; however, many patients with irretractable biventricular failure still require BIVADs as a BTT. Although this need may change, owing to the commercial availability of the Total Artificial Heart (SynCardia Systems Inc, Tucson, Ariz), this device may mimic the effect of BIVADs on the immune system, owing to its larger inner surface areas and chamber valves. Thus, we may continue to see anti-HLA antibody development in our BTT patients who do not qualify for an HMII-only strategy.

The clinical significance of VAD-mediated sensitization is obscured by the fact that significant differences in overall survival and rejection rates have not been realized between VAD-sensitized patients and non-VAD sensitized controls in the posttransplant period. ${ }^{12}$ In addition, our study showed that although presensitized patients had a lower likelihood of being successfully bridged to transplantation, 1-year survival in patients who reach orthotopic heart transplantation is essentially the same in sensitized versus nonsensitized patients. As others have noted, ${ }^{13}$ this finding may be explained by: the fact that virtual cross-matching can enable appropriate donor selection; the efficacy of desensitization protocols; and the fact that 1 year may not be enough time to truly gauge the effects of HLA sensitization on the donor allograft.

Many studies such as this, however, utilized older cytotoxic screens to assess panel reactive antibodies. ${ }^{4,9,12}$ Newer, solid-phase single-antigen bead assays, which have been utilized more recently, have allowed for more-detailed anti-HLA antibody identification and quantification. These newer tests provide significantly more information via quantitative antibody measurements, as documented by mean fluorescence intensity and antibody-specificity assessment.

A review of 565 patients, using traditional lymphocytotoxic methods, found that 14 patients had a positive panel reactive antibody assessment, 5 of whom had donorspecific antibodies. An additional 53 patients were discerned to have HLA antibodies, 14 of whom had donor-specific antibodies. Graft survival of 1 year in the group with antibodies was $42 \%$, compared with $75 \%$ in those patients with no detectable antibodies. ${ }^{14}$ In another study that examined HLA sensitization in the pediatric VAD population, post hoc serum evaluation identified 8 of $19(42 \%)$ patients who were reclassified as sensitized after an initial negative panel reactive antibody evaluation. ${ }^{15}$

In our series, no difference was found in rates of sensitization between the 2 device types, despite differences in fresh

TABLE 3. Maximum mean cumulative HLA antibody strength (MFI) in BIVAD and HMII patients along with mean days to achieve maximum mean cumulative MFI in the 2 groups

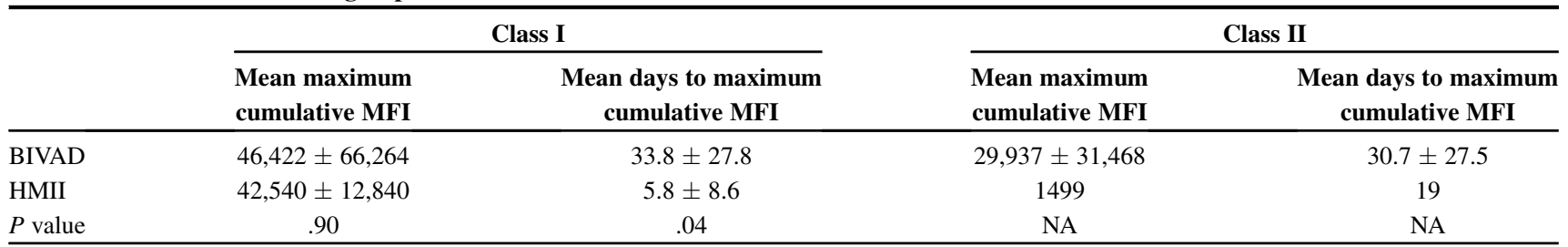

Values are given with \pm SD, as applicable. MFI, Mean fluorescence intensity; $B I V A D$, biventricular assist device; $H M I I$, HeartMate II left ventricular assist device; $N A$, not applicable. 
TABLE 4. Initial and maximum cPRA percentages in patients treated with BIVAD versus HMII

\begin{tabular}{lcccc}
\hline & $\begin{array}{c}\text { Initial } \\
\text { cPRA \% }\end{array}$ & $\begin{array}{c}\text { Maximum } \\
\text { cPRA \% }\end{array}$ & $\begin{array}{c}\text { \% cPRA } \\
\text { change }\end{array}$ & $\begin{array}{c}\text { Days after } \\
\text { VAD }\end{array}$ \\
\hline BIVAD & $16.7 \pm 30.8$ & $50.8 \pm 33.3$ & $34.1 \pm 31.4$ & $20.4 \pm 18.7$ \\
HMII & $48.4 \pm 15.6$ & $52.4 \pm 20.6$ & $4.0 \pm 7.9$ & $19.2 \pm 31.7$ \\
$P$ value & .030 & .920 & .045 & .910 \\
\hline All values are expressed as mean \pm 1 SD. $c P R A$, Calculated panel reactive antibody \\
percentages; $B I V A D$, biventricular assist device; $H M I I$, HeartMate II left ventricular \\
assist device; $V A D$, ventricular assist device.
\end{tabular}

frozen plasma usage. Although the immunomodulatory effects of blood-product transfusion are traditionally attributed to its cellular components, namely leukocytes and platelets, leukocyte depletion has not been shown to reduce sensitization levels in HMII patients. ${ }^{9}$ Cellular blood products have been hypothesized to actually lessen alloimmunization, thereby mitigating the effects of VAD-mediated antibody production in that particular study. ${ }^{9}$ Multivariable analysis controlling for blood-product usage, prior pregnancies, and previous cardiac surgery showed a nearly 3 -fold greater odds ratio for BIVAD-mediated sensitization in our series; however, this difference was not statistically significant.

Only the BIVAD patients, however, developed antibodies in a de novo fashion. Similar to another group, ${ }^{16}$ we found that no HMII patients became newly sensitized after device implantation. Although the strength of antibody expression, as measured by the maximum mean fluorescence intensities, were similar in the 2 groups, the shorter time to antibody production in the HMII recipients was a result of the fact that these patients had immunologic memory to alloantigens at the time of VAD implantation. The breadth of antibody expression, as measured by the calculated panel reactive antibody percentages, was nearly 3 -fold higher in the HMII patients at the onset of therapy. The BIVAD patients, however, did develop equivalent maximum calculated panel reactive antibody levels during VAD support.

Thus, although overall rates of sensitization were not significantly different, the fact that only the BIVAD patients were able to develop previously unexpressed antibodies may indicate the increased sensitizing potential of the larger, pulsatile devices. The mechanistic question remains, however: do BIVADs truly have immunologic properties that lead to the formation of de novo antibodies, or are they merely instigators of inflammation that lead to stimulation of existing memory B cells, thereby creating reexpression of antibodies formed at a previous antigenic exposure. Toll-like receptor ligands, such as HMGB1 (high-mobility group protein B1), are up-regulated during inflammation and have been shown to potentiate the Bcell adaptive immune response by triggering memory $\mathrm{B}$ cells, leading to strong antibody up-regulation. ${ }^{17}$ Determination of whether VADs are truly immunogenic, or rather cause nonspecific up-regulation of polyclonal antibodies via an inflammatory process, will have far-reaching implications, as the therapeutic management for the 2 processes clearly differs.

One limitation of this study is the small number of HMII patients. Despite this liability, however, a clear trend has emerged, of the potential for mechanistic explanations of HLA antibody expression to differ among devices; this finding needs to be confirmed in a larger cohort. The opportunity to further verify mechanisms for VAD-mediated sensitization is currently available via inclusion of newergeneration devices, such as the HeartWare HVAD (HeartWare International Inc, Framingham, Mass) and the Total Artificial Heart.

Another weakness of the study is that non-HLA antibodies were not examined. An accumulating body of literature suggests that antibodies, including those to major histocompatability class I-related chain A-vimentin, heatshock proteins, and cardiac myosin-additionally have detrimental effects on allograft function. ${ }^{18}$ In a study of pediatric patients bridged with BIVAD support, $33 \%$ of patients showed sensitization by enzyme-linked immunosorbant assay (ELISA) that was not corroborated by Luminex studies (Gen-Probe). This finding indicated that non-HLA antibodies may represent a component of sensitization that should be characterized, as it would identify a particularly high-risk group for primary graft dysfunction. ${ }^{19}$ Future studies in this important area are needed, to discern whether the 2 device populations differ in nonHLA antibody expression.

\section{CONCLUSIONS}

Although no differences were found between the BIVAD and HMII groups, in either rates of patients who expressed anti-HLA antibodies, or class I and II expression, key findings suggest that the older, pulsatile devices had greater sensitization potential. Namely, the fact that only the former group was able to develop antibodies in a de novo fashion after device implantation, whereas the latter merely heightened existing antibody levels, suggests that differing mechanisms of action were at work. Ultimately, characterization of the antibody response in VAD patients will enhance our understanding of the process of sensitization in the pretransplant patient in general.

Unlike the other sources of antigenic stimulation, such as pregnancy and blood-product exposure, VADs remain in place during the therapeutic interval and provide an ongoing antigenic or inflammatory stimulus. The fact that the stimulation is continuous may influence whether desensitization protocols truly alter the pretransplant patient's immunologic state or merely transiently reduce circulating antibodies for a brief period of time. By defining the mechanism of sensitization in VAD patients, we can better elucidate the best therapies that can be used to lower circulating 
antibodies, through identification of those patients who are most at risk and most amenable to treatment protocols.

\section{References}

1. Kobashigawa J, Mehra M, West L, Kerman R, George J, Rose M, et al. Report from a consensus conference on the sensitized patient awaiting heart transplantation. J Heart Lung Transplant. 2009;28:213-25.

2. Michaels PJ, Espejo ML, Kobashigawa J, Alejos JC, Burch C, Takemoto S, et al. Humoral rejection in cardiac transplantation: risk factors, hemodynamic consequences and relationship to transplant coronary artery disease. J Heart Lung Transplant. 2003;22:58-69.

3. Reed EF, Demetris AJ, Hammond E, Itescu S, Kobashigawa JA, Reinsmoen NL, et al. Acute antibody-mediated rejection of cardiac transplants. J Heart Lung Transplant. 2006;25:153-9.

4. George I, Colley P, Russo MJ, Martens TP, Burke E, Oz MC, et al. Association of device surface and biomaterials with immunologic sensitization after mechanical support. J Thorac Cardiovasc Surg. 2008;135:1372-9.

5. Malickaite R, Rucinskas K, Staneviciene A, Miniauskas S, Maneikiene V, Zuoziene G, et al. Sensitization and post-transplant course after the implantation of ventricular assist device. Interact Cardiovasc Thorac Surg. 2009;8:339-42.

6. Thompson LO, Loebe M, Noon GP. What price support? Ventricular assist device induced systemic response. ASAIO J. 2003;49:518-26.

7. Blumberg JM, Gritsch HA, Reed EF, Cecka JM, Lipshutz GS, Danovitch GM, et al. Kidney paired donation in the presence of donor-specific antibodies. Kidney Int. 2013;84:1009-16.

8. John R, Lietz K, Schuster M, Naka Y, Rao V, Mancini DM, et al. Immunologic sensitization in recipients of left ventricular assist devices. J Thorac Cardiovasc Surg. 2003;125:578-91.

9. Drakos SG, Stringham JC, Long JW, Gilbert EM, Fuller TC, Campbell BK, et al Prevalence and risks of allosensitization in HeartMate left ventricular assist device recipients: the impact of leukofiltered cellular blood product transfusions. $J$ Thorac Cardiovasc Surg. 2007;133:1612-9.
10. Itescu S, John R. Interactions between the recipient immune system and the left ventricular assist device surface: immunological and clinical implications. Ann Thorac Surg. 2003;75:S58-65.

11. Schuster M, Kocher A, John R, Hoffman M, Ankersmit J, Lietz K, et al. B-cell activation and allosensitization after left ventricular assist device implantation is due to T-cell activation and CD40 ligand expression. Hum Immunol. 2002; 63:211-20.

12. Joyce DL, Southard RE, Torre-Amione G, Noon GP, Land GA, Loebe M. Impact of left ventricular assist device (LVAD)-mediated humoral sensitization on posttransplant outcomes. J Heart Lung Transplant. 2005;24:2054-9.

13. Shankar N, Daly R, Geske J, Kushwaha SK, Timmons M, Joyce L, et al. LVAD implant as a bridge to heart transplantation is associated with allosensitization as measured by single antigen bead assay. Transplantation. 2013;96:324-30.

14. Smith JD, Hamour IM, Banner NR, Rose ML. C4d fixing, luminex binding antibodies - a new tool for prediction of graft failure after heart transplantation. Am J Transplant. 2007;7:2809-15.

15. Yang J, Schall C, Smith D, Kreuser L, Zamberlan M, King K, et al. HLA sensitization in pediatric pre-transplant cardiac patients supported by mechanical assist devices: the utility of Luminex. J Heart Lung Transplant. 2009;28:123-9.

16. Drakos SG, Kfoury AG, Kotter JR, Reid BB, Clayson SE, Selzman CH, et al. Prior human leukocyte antigen-allosensitization and left ventricular assist device type affect degree of post-implantation human leukocyte antigen-allosensitization. J Heart Lung Transplant. 2009;28:838-42.

17. Bernasconi NL, Onai N, Lanzavecchia A. A role for Toll-like receptors in acquired immunity: up-regulation of TLR9 by BCR triggering in naive B cells and constitutive expression in memory B cells. Blood. 2003;101:4500-4.

18. Smith JD, Hamour IM, Burke MM, Mahesh B, Stanford RE, Haj-Yahia S, et al. A reevaluation of the role of IgM non-HLA antibodies in cardiac transplantation. Transplantation. 2009;87:864-71.

19. Gandhi SK, Huddleston CB, Balzer DT, Epstein DJ, Boschert TA, Canter CE. Biventricular assist devices as a bridge to heart transplantation in small children. Circulation. 2008;118:S89-93.

\section{EDITORIAL COMMENTARY}

\section{De novo allosensitization only seen with biventricular assist device support}

Stephen H. McKellar, MD, MSc

See related article on pages 1161-6.

Allosensitization of patients receiving mechanical circulatory support (MCS) is an important topic, because

From the Division of Cardiothoracic Surgery, University of Utah School of Medicine, Salt Lake City, Utah.

Disclosures: Author has nothing to disclose with regard to commercial support.

Received for publication Jan 15, 2015; accepted for publication Jan 15, 2015; available ahead of print Feb 7, 2015.

Address for reprints: Stephen H. McKellar, MD, MSc, Division of Cardiothoracic Surgery, University of Utah School of Medicine, 30 North 1900 East, 3C127 SOM, Salt Lake City, UT 84132 (E-mail: stephen.mckellar@hsc.utah.edu).

J Thorac Cardiovasc Surg 2015;149:1166-7

$0022-5223 / \$ 36.00$

Copyright (c) 2015 by The American Association for Thoracic Surgery

http://dx.doi.org/10.1016/j.jtcvs.2015.01.025 many patients are receiving support as a bridge to cardiac transplant. In this issue of the Journal, Kwon and coauthors $^{1}$ present a review of their institutional experience with 68 patients receiving MCS, in the form of either a left ventricular assist device (LVAD) or biventricular assist devices (BIVADs). They observed that the type of MCS did not predict allosensitization but that for patients with LVAD support to become sensitized, they required previous sensitization. In contrast, de novo sensitization only occurred in patients supported with BIVADs. Kwon and coauthors ${ }^{1}$ concluded that de novo sensitization with BIVAD support occurs through an undefined mechanism separate from the worsening, preexisting sensitization in the patients with LVAD support.

Although the issue has been discussed for years, the clinical implication of MCS-related allosensitization is 